\title{
Histamine Receptor Antagonists, Loratadine and Azelastine, Sensitize P-gp-overexpressing Antimitotic Drug-resistant KBV20C Cells Through Different Molecular Mechanisms
}

\author{
JI YEONG KIM, KYEONG SEOK KIM, IN SU KIM and SUNGPIL YOON \\ School of Pharmacy, Sungkyunkwan University, Suwon, Republic of Korea
}

\begin{abstract}
Background/Aim: Previously, we showed that KBV20C cancer cells highly resistant to antimitotic drugs were sensitized by co-treatment with a repositioned drug fluphenazine. Materials and Methods: Considering that fluphenazine plays a role as a histamine receptor antagonist, we investigated low doses of 21 other histamine receptor antagonists (lidocaine, cimetidine, chlorpromazine, diphenhydramine, promethazine, ranitidine, famotidine, clemastine, chlorpheniramine, desloratadine, loratadine, cyproheptadine, azelastine, brompheniramine, carbinoxamine, fexofenadine, hydroxyzine, levocetirizine, meclizine, nizatidine, and pemirolast) to identify repositioned drugs for their sensitizing effects on antimitotic drug-resistant KBV20C cells at relatively low doses. Results: Co-treatment with loratadine, and with azelastine highly sensitized KBV20C cells to vincristine treatment. Loratadine and azelastine reduced cell viability, increased $G_{2}$ arrest, and up-regulated apoptosis when coadministered with vincristine. In detailed quantitative analysis, combination of vincristine with loratadine had a higher sensitization effect than that with azelastine. Azelastine had a higher P-glycoprotein (P-gp)-inhibitory activity, similar to that of verapamil, indicating that sensitization by vincristine-azelastine involved the P-gp-inhibitory effects of azelastine. However, loratadine had a very low P-gpinhibitory activity, suggesting that loratadine sensitization to vincristine is independent of the P-gp-inhibition. Cotreatment with eribulin and loratadine increased the sensitization of KBV2OC cells, suggesting that loratadine can be combined with other antimitotic drugs to sensitize resistant cancer cells. Conclusion: These findings provide important
\end{abstract}

Correspondence to: Sungpil Yoon, Ph.D., School of Pharmacy, Sungkyunkwan University, 2066 Seobu-ro, Jangan-gu, Suwon, Gyeonggi-do, 16419, Republic of Korea. Tel: +82 1055024893, Fax: +82 312928800, e-mail: syoon88@gmail.com

Key Words: Loratadine, azelastine, histamine receptor antagonists, repositioning drug, Cancer, P-gp, drug-resistance. information regarding the sensitization of drug-resistant cells and indicate that loratadine may be used in patients with potentially resistant cancer without any toxic effects from $P$ gp inhibition.

Antimitotic drugs act by preventing polymerization or depolymerization of the microtubules. Paclitaxel, docetaxel, vincristine, vinorelbine, vinblastine, and eribulin show this type of activity (1-4). Although antimitotic drugs are widely used in the treatment of cancer, cancer cells can develop resistance in various ways. P-glycoprotein (P-gp) overexpression is a main mechanism of resistance to antimitotic drugs. P-gp is a membrane channel protein that can pump out antimitotic drugs in order to avoid druginduced toxicity (5-8). Sensitization of cancer cells against overexpression of P-gp can lead to better treatment of tumors which develop resistance to antimitotic drugs. Although P-gp inhibitors have been developed, their toxicity to normal cells leads to treatment failure. Therefore, it is important to investigate novel therapeutic options without $\mathrm{P}$-gp inhibition for P-gp-overexpressing drug-resistant cancer.

In this study, we aimed at identifying novel repositioned drugs for their sensitizing efficacy in P-gp-overexpressingresistant cancer cells when used in combination with antimitotic drugs and to investigate the mechanisms involved. The need for novel treatments against P-gp-overexpressing resistant cancer cells can be effectively addressed provided that repositioned drugs are identified since these drugs would not need further toxicity evaluation (9-11).

In previous studies, we demonstrated that the histamine receptor antagonist fluphenazine has $\mathrm{P}$-gp-inhibitory activity and drug-sensitization effects on P-gp-overexpressing drugresistant cancer cells $(12,13)$. In addition, histamine receptor antagonists have also been shown to sensitize drug-resistant cancer cells (14-16). However, comparison of individual histamine receptor antagonists and their exact mechanisms of action have not yet been investigated.

In this study, we investigated different histamine receptor antagonists for their sensitizing effects on drug-resistant 
cancer cells. Based on our literature search, we identified 21 histamine receptor antagonists, namely, lidocaine, cimetidine, chlorpromazine, diphenhydramine, promethazine, ranitidine, famotidine, clemastine, chlorpheniramine, desloratadine, loratadine, cyproheptadine, azelastine, brompheniramine, carbinoxamine, fexofenadine, hydroxyzine, levocetirizine, meclizine, nizatidine, and pemirolast. We then investigated which histamine receptor antagonists have a relatively low half-maximal inhibitory concentration $\left(\mathrm{IC}_{50}\right)$ which sensitized P-gp-overexpressing drug-resistant KBV20C cancer cells. We also studied the mechanisms involved in the sensitization of resistant cancer cells.

As these drugs are used in clinical settings as anti-allergic drugs, these results can contribute to the development of histamine receptor antagonist-based therapy in the cotreatment of highly drug-resistant tumors.

\section{Materials and Methods}

Reagents and cell culture. Rhodamine123 (rhodamine), fluphenazine, and verapamil were purchased from Sigma-Aldrich (St. Louis, MO, USA). Vincristine was purchased from Enzo Life Sciences (Farmingdale, NY, USA). Lidocaine, cimetidine, chlorpromazine, diphenhydramine, promethazine, ranitidine, famotidine, clemastine fumarate, chlorpheniramine maleate, desloratadine, loratadine, cyprophetadine, azelastine, brompheniramine meleate, carbinoxamine maleate, fexofenadine, hydroxyzine dihydrochloride, levocetirizine dihydrochloride, meclizine dihydrochloride, nizatidine, and pemirolast potassium were purchased from Selleckchem (Houston, TX, USA). Aqueous solutions of eribulin (halaven; Eisai Korea, Seoul, South Korea) were obtained from the National Cancer Center in South Korea

Human oral squamous carcinoma cell line, $\mathrm{KB}$, and its multidrug-resistant subline, KBV20C, were obtained from Dr. Yong Kee Kim (College of Pharmacy, Sookmyung Women's University, Seoul, South Korea) and have been previously described $(13,17-$ 20). All cell lines were cultured in RPMI 1640 containing $10 \%$ fetal bovine serum, $100 \mathrm{U} / \mathrm{ml}$ penicillin, and $100 \mu \mathrm{g} / \mathrm{ml}$ streptomycin (WelGENE, Daegu, South Korea).

Microscopic observation. Cells were grown to $40-50 \%$ confluence in $60-\mathrm{mm}$ diameter dishes and treated with $5 \mu \mathrm{M}$ clemastine, loratadine, azelastine or fluphenazine, or $10 \mu \mathrm{M}$ verapamil alone and in combination with $60 \mathrm{nM}$ eribulin or $5 \mathrm{nM}$ vincristine for $24 \mathrm{~h}$ or $48 \mathrm{~h}$. The medium was removed, and phosphate-buffered saline (PBS) was added into each dish. Attached cells were examined immediately in two independent experiments using an ECLIPSE Ts2 inverted routine microscope (Nikon, Tokyo, Japan) with a $4 \times$ or a $10 \times$ objective lens (Nikon's Microscopy U).

Rhodamine uptake tests. Inhibition of $\mathrm{P}$-gp was assessed by a previously described method $(13,17-20)$. Briefly, cells were grown to $40-50 \%$ confluence in $60-\mathrm{mm}$ diameter dishes and treated with 5 $\mu \mathrm{M}$ clemastine, loratadine or azelastine, or $10 \mu \mathrm{M}$ verapamil for 4 $\mathrm{h}$ or $24 \mathrm{~h}$ at $37^{\circ} \mathrm{C}$. Cells were then incubated with $2 \mu \mathrm{g} / \mathrm{ml}$ rhodamine for $1 \mathrm{~h} 30 \mathrm{~min}$ at $37^{\circ} \mathrm{C}$. The medium was removed, the cells were washed with PBS, and stained cells were then analyzed in two independent experiments using a Guava EasyCyte Plus Flow Cytometer (Merck Millipore, Burlington, MA, USA).
Fluorescence-activated cell sorting (FACS) analysis. FACS analysis was performed as previously described $(13,17-20)$. Cells were grown to $40-50 \%$ confluence in $60-\mathrm{mm}$ diameter dishes and treated with $5 \mu \mathrm{M}$ clemastine, loratadine or azelastine, or $10 \mu \mathrm{M}$ verapamil alone and in combination with $5 \mathrm{nM}$ vincristine for $24 \mathrm{~h}$. The cells were then detached by trypsin and pelleted by centrifugation. The pelleted cells were washed thoroughly with PBS, suspended in $75 \%$ ethanol for at least $1 \mathrm{~h}$ at $4^{\circ} \mathrm{C}$, washed with PBS, and re-suspended in a cold propidium iodide (PI) staining solution $(100 \mu \mathrm{g} / \mathrm{ml}$ RNase A and $50 \mu \mathrm{g} / \mathrm{ml} \mathrm{PI} \mathrm{in} \mathrm{PBS)} \mathrm{for} 30 \mathrm{~min}$ at $37^{\circ} \mathrm{C}$. The stained cells were analyzed in two independent experiments for relative DNA content using a Guava EasyCyte Plus Flow Cytometer (Merck Millipore, Burlington, MA, USA).

Annexin $V$ analysis. Annexin $\mathrm{V}$ analysis was performed by using the annexin V-fluorescein isothiocyanate (FITC) staining kit (BD Bioscience, Franklin, NJ, USA) as previously described (13, 17-20). Cells were grown to $40-50 \%$ confluence in $60-\mathrm{mm}$ diameter dishes and treated with $5 \mu \mathrm{M}$ clemastine, loratadine, or azelastine alone and in combination with $5 \mathrm{nM}$ vincristine for $24 \mathrm{~h}$. The cells were then detached by trypsin and pelleted by centrifugation. The pelleted cells were washed with PBS. Cells in $100 \mu \mathrm{l}$ of binding buffer received $5 \mu \mathrm{l}$ of Annexin V-FITC and $5 \mu \mathrm{l}$ of PI and were then incubated for $15 \mathrm{~min}$ at room temperature. The stained cells were analyzed in two independent experiments using a Guava EasyCyte Plus Flow Cytometer (Merck Millipore, Burlington, MA, USA).

Cell viability assay. Cell proliferation was estimated by a colorimetric assay using the EZ-CyTox cell viability assay kit (Daeillab, South Korea) according to the manufacturer's instructions. Briefly, cells were grown to $30-40 \%$ confluence in wells of 96-well plates and treated with $5 \mu \mathrm{M}$ of lidocaine, cimetidine, chlorpromazine, diphenhydramine, promethazine, ranitidine, famotidine, clemastine, chlorpheniramine, desloratadine, loratadine, cyproheptadine, azelastine, brompheniramine, carbinoxamine, fexofenadine, hydroxyzine, levocetirizine, meclizine, nizatidine, pemirolast, or $10 \mu \mathrm{M}$ verapamil alone and in combination with $5 \mathrm{nM}$ vincristine for $48 \mathrm{~h}$. They were then incubated with $10 \mu \mathrm{l}$ of EZ-CyTox solution for 1-2 $\mathrm{h}$ at $37^{\circ} \mathrm{C}$. Absorbance at $450 \mathrm{~nm}$ was determined immediately using a VERSA MAX Microplate Reader (Molecular Devices Corp., Sunnyvale, CA, USA). All experiments were performed at least in triplicate and repeated twice.

Statistical analysis. Data are presented as mean \pm standard deviation (S.D.). Statistical analysis was performed using Student's $t$-test and one-way analysis of variance (ANOVA) followed by a multiplecomparison test. Results were considered statistically significant compared to those of the control when $p<0.05$.

\section{Results}

Loratadine and azelastine sensitized resistant KBV20C cancer cells to vincristine treatment better than other histamine receptor antagonists. We aimed at identifying drugs that might be repositioned to sensitize resistant cells or enhance the efficacy of other agents when used in combination. Previously, we demonstrated that the histamine receptor antagonist fluphenazine had P-gp-inhibitory activity and increased the sensitivity of resistant cancer cells to antimitotic drugs $(12,13)$. 

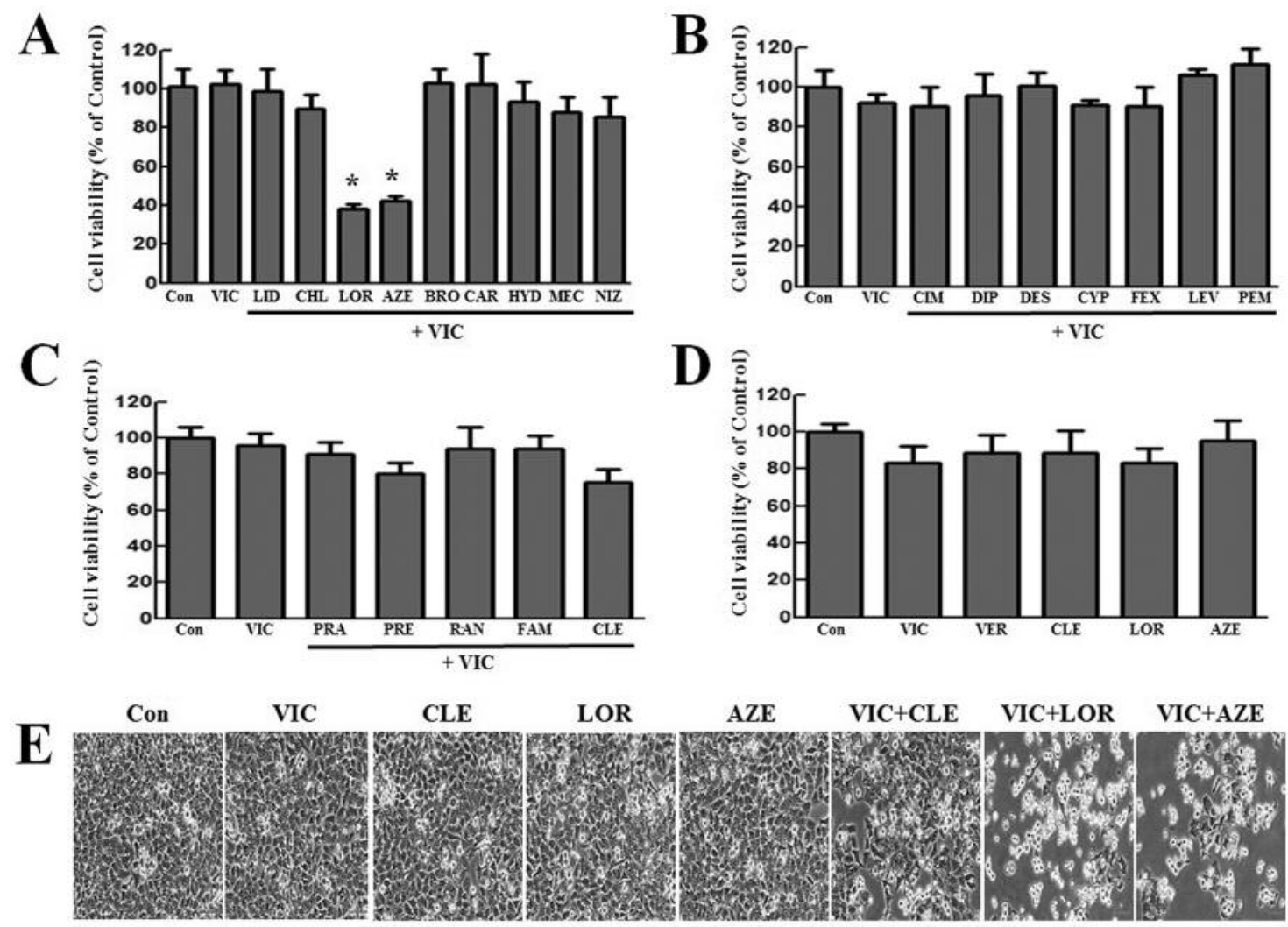

Figure 1. Loratadine and azelastine sensitize resistant KBV20C cancer cells to vincristine (VIC) better than other histamine receptor antagonists. A-C: KBV20C cells were plated on 96-well plates and grown to 30-40\% confluence. The cells were then treated for $48 \mathrm{~h}$ with $5 \mu \mathrm{M}$ of lidocaine (LID), chlorpheniramine (CHL), loratadine (LOR), azelastine (AZE), brompheniramine (BRO), carbinoxamine (CAR), hydroxyzine (HYD), meclizine (MEC), nizatidine (NIZ) (A), cimetidine (CIM), diphenhydramine (DIP), desloratadine (DES), cyproheptadine (CYP), fexofenadine (FEX), levocetirizine (LEV), pemirolast (PEM) (B), chlorpromazine (PRA), promethazine (PRE), ranitidine (RAN), famotidine (FAM), or clemastine (CLE) (C) alone and in combination with $5 \mathrm{nM}$ vincristine (VIC), or with $0.1 \%$ dimethyl sulfoxide (Con). Cell viability assay was performed as described in the Materials and Methods. The data are presented as the mean \pm S.D. of at least two experiments repeated in triplicate. Statistical analysis was conducted using one-way analysis of variance (ANOVA) followed by multiple-comparison test. *Significantly different at $p<0.05$ compared to the corresponding control. D: KBV20C cells were plated on 96-well plates and grown to 30-40\% confluence. The cells were then treated for $48 \mathrm{~h}$ with $5 \mathrm{nM}$ vincristine, clemastine, loratadine, or azelastine, or $10 \mu \mathrm{M}$ verapamil, or 0.1\% DMSO (Con). Cell viability assay was performed as described in the Materials and Methods. E: KBV20C cells were grown on $60 \mathrm{~mm}$-diameter dishes and treated with $5 \mathrm{nM}$ clemastine, loratadine, or azelastine, alone or combined with $5 \mathrm{nM}$ vincristine, or with $0.1 \%$ DMSO (Con). After 1 day, cells were observed using an inverted microscope at $\times 10$ magnification (scale bar=100 $\mu \mathrm{m}$ ).

Considering that fluphenazine is a histamine receptor antagonist, we planned to identify other histamine receptor antagonists for sensitizing resistant cancer cells at relatively low doses. Therefore, we performed further detailed analysis with 21 known histamine receptor antagonists lidocaine, cimetidine, chlorpromazine, diphenhydramine, promethazine, ranitidine, famotidine, clemastine, chlorpheni-ramine, desloratadine, loratadine, cyproheptadine, azelastine, brompheniramine, carbinoxamine, fexofenadine, hydroxyzine, levocetirizine, meclizine, nizatidine, and pemirolast. We tested sensitivity to vincristine, an antimitotic drug that is routinely used as a chemotherapeutic agent in cancer $(21,22)$. KBV20C resistant cancer cells have a vincristine-resistant phenotype due to P-gp overexpression $(13,19,20)$.
Firstly, we performed quantitative analysis with a cell viability test. As seen in Figure $1 \mathrm{~A}-\mathrm{C}$, loratadine and azelastine highly reduced viability of vincristine-treated KBV20C cells. Cell viability with vincristine co-treatment with loratadine and with azelastine was $>60 \%$ as compared to the control. There was no difference between the control and individual treatments with loratadine or azelastine (Figure 1D), suggesting that sensitization by co-treatment resulted in synergistic effects of loratadine or azelastine in vincristine-treated cancer cells.

We confirmed the results of viability tests by microscopic observation. As shown in Figure 1E, $5 \mu \mathrm{M}$ of loratadine or azelastine sensitized cells to vincristine, whereas no effect was observed on individual treatment with loratadine or 

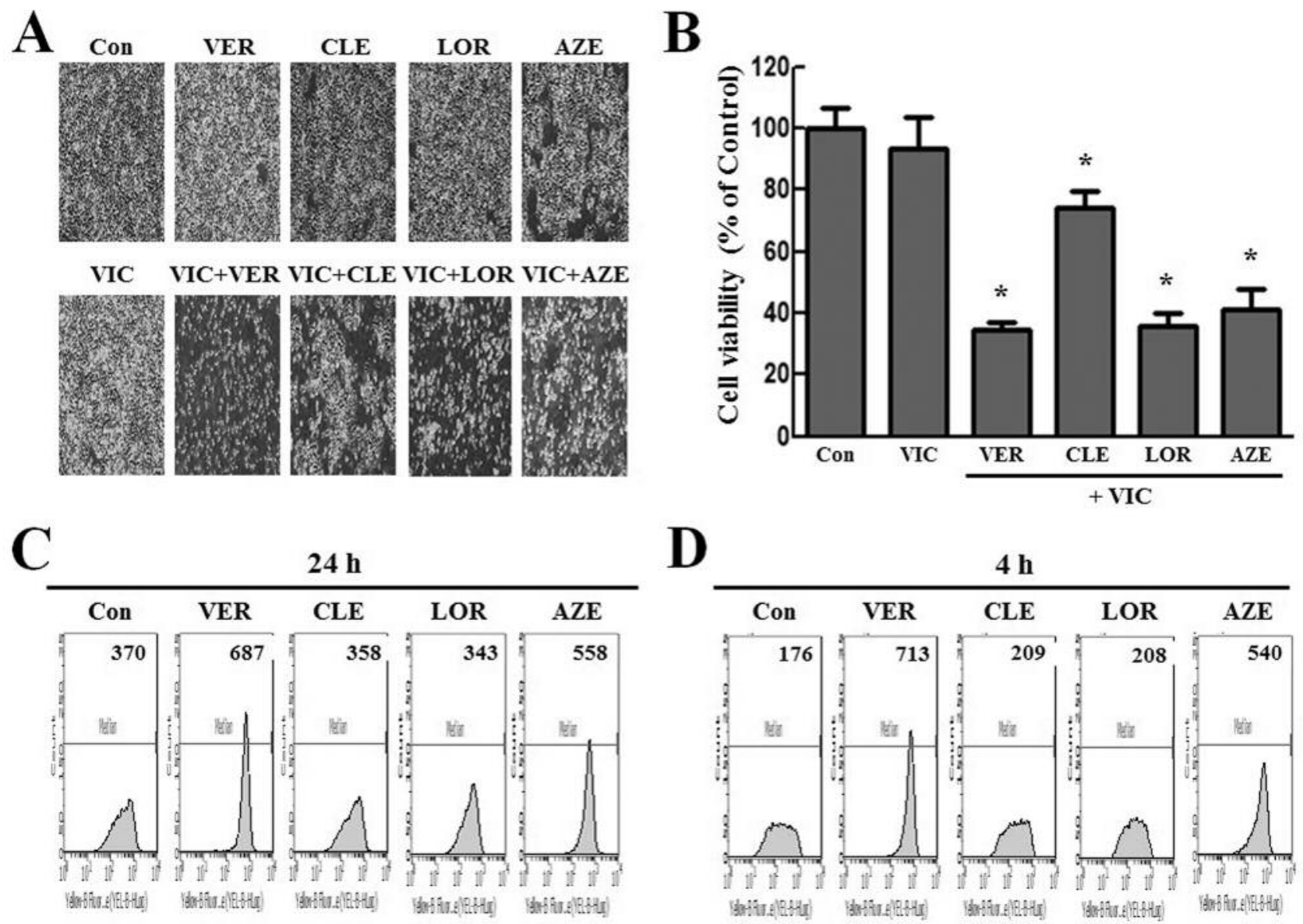

Figure 2. Loratadine has a low P-glycoprotein (P-gp)-inhibitory activity, whereas azelastine has high P-gp-inhibitory activity. A: KBV20C cells were grown on $60 \mathrm{~mm}$-diameter dishes and treated with $5 \mu \mathrm{M}$ clemastine (CLE), loratadine (LOR), azelastine (AZE), or $10 \mu \mathrm{M}$ verapamil (VER), alone or combined with $5 \mathrm{nM}$ vincristine (VIC), or $0.1 \%$ DMSO (Con). After 1 day, all cells were observed using an inverted microscope at $\times 4$ magnification (scale bar=100 $\mu \mathrm{m}$ ). B: KBV20C cells were plated on 96-well plates and grown to 30-40\% confluence. The cells were then treated for $48 \mathrm{~h}$ with as above. Cell viability assay was performed as described in the Materials and Methods. C and D: KBV20C cells were grown on $60 \mathrm{~mm}$-diameter dishes and treated with single agents as above. After 24 or $4 \mathrm{~h}$, cells were stained with rhodamine and examined by using FACS analysis, as described in the Materials and Methods. Data are presented as the mean \pm S.D.

azelastine. Further detailed analysis of both viability tests and microscopic results showed that co-treatment with vincristine-clemastine slightly increased sensitization (Figure $1 \mathrm{C}$ and $\mathrm{E})$.

Altogether, when we analyzed 21 known histamine receptor antagonists to identify novel repositioned drugs, we observed that both loratadine and azelastine at a low dose had high sensitization effects, reduced viability more than the other drugs. We conclude that low dose of loratadine and azelastine can be used to reduce drug toxicity and sensitize vincristine-resistant cancer cells.

Loratadine shows low P-gp-inhibitory activity, whereas azelastine shows high P-gp-inhibitory activity. Next, we compared the effects of loratadine and azelastine on sensitizing cells to verapamil (positive control) which is a Pgp inhibitor (5). It is well-known that co-treatment with verapamil increases sensitization of KBV20C cells to vincristine $(18,23)$. As seen in Figure $2 \mathrm{~A}$, compared to $10 \mu \mathrm{M}$ verapamil, $5 \mu \mathrm{M}$ of loratadine or azelastine produced similar sensitization combined with vincristine of cells. This suggests that a low dose of loratadine or azelastine is adequate and as effective as the P-gp inhibitor verapamil in sensitizing P-gpoverexpressing resistant cancer cells.

We performed a more detailed quantitative analysis with cellular viability test. Although there was no reduced viability after treatment with individual drugs (Figure 1D), we found that loratadine and azelastine highly reduced viability of vincristine-treated resistant KBV20C cells, as much as did verapamil (Figure 2B). The results demonstrated 
A
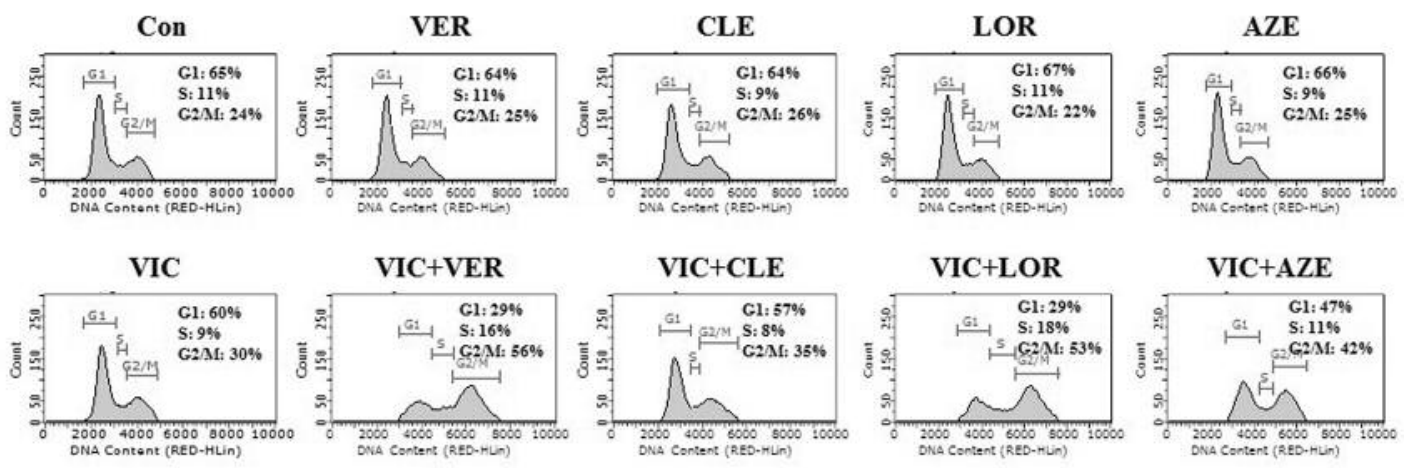

B
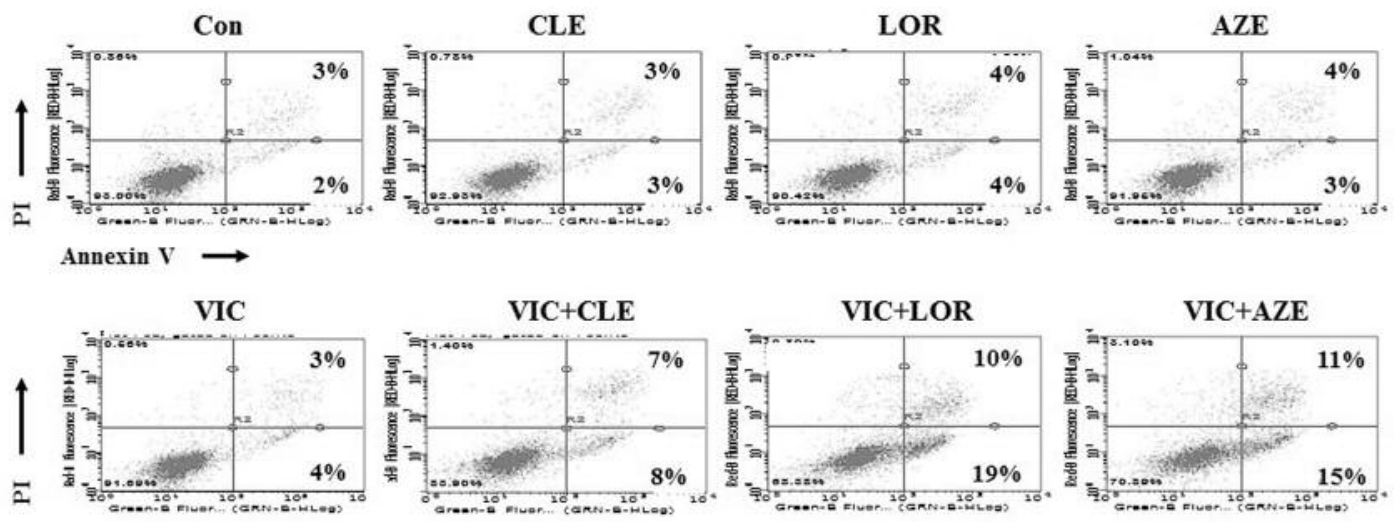

Annexin V

Figure 3. Loratadine highly sensitizes resistant KBV20C cells to vincristine via up-regulation of apoptosis through induction of $G_{2}$ arrest. A: KBV20C cells were grown on 60-mm-diameter dishes and treated with $5 \mu \mathrm{M}$ clemastine (CLE), loratadine (LOR), azelastine (AZE), or $10 \mu \mathrm{M}$ verapamil (VER), alone or in combination with $5 \mathrm{nM}$ vincristine, or with $0.1 \%$ DMSO (Con). After $24 \mathrm{~h}$, annexin V analyses were performed as described in the Materials and Methods. B: KBV20C cells were grown on 60 mm-diameter dishes and treated as above. After 24 h, FACS analyses were performed as described in the Materials and Methods. Data are presented as the mean \pm S.D.

that both loratadine and azelastine are as effective as verapamil in sensitizing vincristine-resistant cancer cells. However, as expected, vincristine-clemastine co-treatment had low sensitization effect as compared to that of vincristine combined with verapamil, loratadine, or azelastine (Figure $2 \mathrm{~A}$ and $\mathrm{B}$ ).

Next, we tested the P-gp-inhibitory activities of clemastine, loratadine, and azelastine in P-gp-overexpressing KBV20C cells because we assumed that the difference in the degree of $\mathrm{P}$-gp inhibition among histamine receptor antagonists is responsible for the difference in sensitivity of KBV20C cells to combined treatment with vincristine. However, as shown in Figure 2C and D, P-gp inhibition by loratadine was much lower than that by the well-known P-gp inhibitor verapamil and was also similar to the blank control. Clemastine also showed less P-gp-inhibitory activity, similarly to loratadine. Sensitivity to vincristine-loratadine was much higher than that to vincristine-clemastine (Figure $2 \mathrm{~A}$ and $2 \mathrm{~B}$ ) indicating that vincristine-loratadine sensitization of KBV20C cells was independent of the P-gp-inhibitory effects of loratadine. When we analyzed the P-gp-inhibitory activities of azelastine, we found that it had a similar P-gp-inhibitory activity as that of verapamil suggesting that $\mathrm{P}-\mathrm{gp}$ inhibition by azelastine plays a major role in the sensitization to co-treatment with vincristine. As shown in Figure $2 \mathrm{C}$ and $\mathrm{D}$, treatment with azelastine or verapamil for $4 \mathrm{~h}$ gave similar results to those obtained after $24 \mathrm{~h}$ of treatment. This suggests that azelastine inhibited P-gp by direct binding, similar to the mechanism involved in inhibition by verapamil. It appears that azelastine can be used to replace well-known P-gp inhibitors as it may inhibit P-gp with reduced toxicity in clinical settings.

Collectively, we found that the histamine receptor antagonists have different $\mathrm{P}$-gp-inhibitory activities and function differently in sensitizing P-gp-overexpressing KBV20C cells to vincristine. Although vincristine-loratadine and vincristine-azelastine co-treatments had similar effects on P-gp overexpressing KBV20C cells, interestingly, loratadine sensitized KBV20C cells to vincristine inducing 


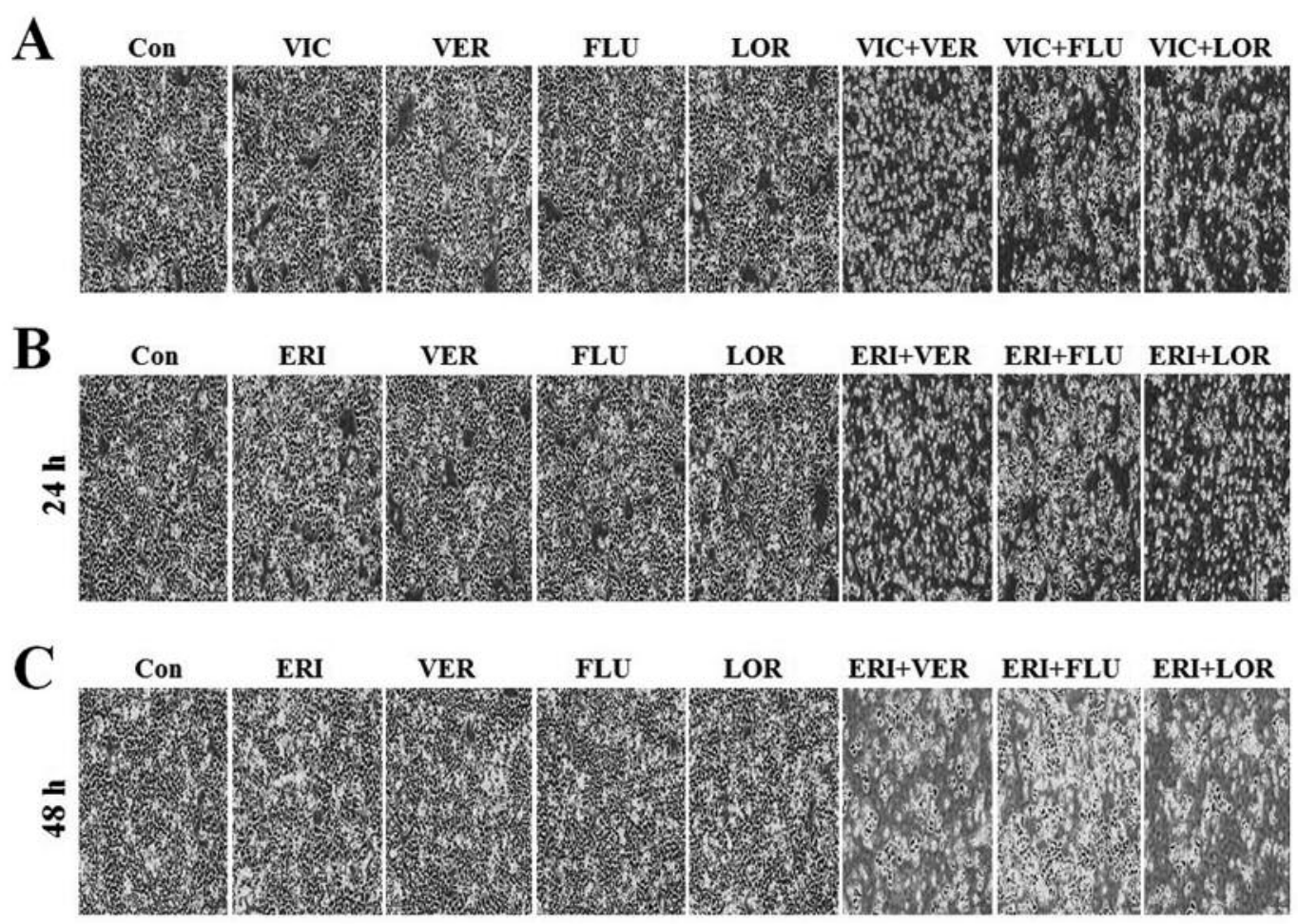

Figure 4. Loratadine increases sensitization of KBV20C cells to antimitotic drugs at low doses. A: KBV20C cells were grown on 60 mm-diameter dishes and treated with $5 \mu M$ fluphenazine (FLU), $5 \mu M$ loratadine (LOR), $10 \mu M$ verapamil (VER), or 5 nM vincristine (VIC), alone or in combination, or with $0.1 \%$ DMSO (Con). After 1 day, cells were observed using an inverted microscope at $\times 4$ magnification (scale bar=100 $\mu$ m). $B$ and C: KBV20C cells were grown on $60 \mathrm{~mm}$-diameter dishes and treated with $60 \mathrm{nM}$ eribulin (ERI), $10 \mu \mathrm{M}$ verapamil, $5 \mu \mathrm{M}$ fluphenazine, $5 \mu \mathrm{M}$ loratadine, alone or in combination, or 0.1\% DMSO (Con). After 24 or $48 \mathrm{~h}$, cells were observed using an inverted microscope at $\times 4$ magnification (scale bar $=100 \mu \mathrm{m})$. Data are presented as the mean \pm S.D.

very low P-gp-inhibitory activity, whereas azelastine sensitized them with high P-gp-inhibitory activity. Therefore, we conclude that vincristine-loratadine and vincristineazelastine co-treatments have different mechanisms of sensitization of KBV20C cells.

Vincristine combined with loratadine sensitizes KBV20C cells via apoptosis through induction of $G_{2}$ arrest. In order to further clarify the mechanism of action of histamine receptor antagonist co-treatments with vincristine, we performed FACS analyses. As shown in Figure 3A, vincristine combined with loratadine, and with azelastine considerably increased the number of cells in $\mathrm{G}_{2}$ arrest compared to that observed after individual treatment with either agent. Vincristine combined with clemastine also increased $G_{2}$ arrest to a small extent, suggesting that clemastine also had low synergistic effects. This indicates that facilitation of cell-cycle arrest resulted in the reduction of cellular viability.

When $\mathrm{G}_{2}$ arrest was quantitatively estimated, we found that the proportion of $\mathrm{G}_{2}$-arrested cells was approximately
$35 \%$ for vincristine with clemastine, $53 \%$ when combined with loratadine, and $42 \%$ when combined with azelastine (Figure 3A). Vincristine-verapamil co-treatment as a positive control also resulted in $56 \% \mathrm{G}_{2}$-arrested cells (Figure $3 \mathrm{~A}$ ). The results suggest that the effect of vincristine-loratadine co-treatment in causing $G_{2}$ arrest was greater than that of vincristine-azelastine and was similar to that of vincristineverapamil.

Using annexin $\mathrm{V}$ analysis, we also tested whether vincristine combined with histamine receptor antagonists increased cell death by apoptosis. As seen in Figure 3B, apoptotic cell death largely increased after co-treatments of vincristine with loratadine and with azelastine, whereas a small increase in apoptotic cell death was detected with vincristine-clemastine. We assumed that reduced $\mathrm{G}_{2}$ arrest contributes to increased apoptotic death. Annexin V staining was also analyzed in more detail. As seen in Figure 3B, the proportion of apoptotic cells in both early and late phases after treatment of vincristine was about $15 \%$ when combined with clemastine, $29 \%$ combined with loratadine, and $26 \%$ with azelastine, suggesting that vincristine-loratadine sensitizes the cells much better than 
vincristine-clemastine and vincristine-azelastine. Overall, among the histamine receptor antagonists, co-treatment with loratadine highly augmented the sensitization of resistant KBV20C cells to vincristine via $\mathrm{G}_{2}$ cell-cycle arrest and apoptosis. Considering that loratadine sensitized KBV20C cells to vincristine with very low P-gp-inhibitory activity (Figure 2C and D), it may be useful in clinical settings due to minimal toxic P-gp-inhibitory activity in normal cells.

Loratadine and fluphenazine increased sensitization of KBV20C cells to antimitotic drug at a low dose. The histamine receptor antagonist fluphenazine has been demonstrated to sensitize KBV20C cells to vincristine and can be used as a repositioned drug $(12,13,19)$. We tested whether the combination of vincristine with loratadine would be more effective than co-treatment with fluphenazine. Microscopic observations indicated that both co-treatments showed sensitization effects similar to vincristine-verapamil (Figure 4A). We concluded that highly drug-resistant KBV20C cells can be sensitized by co-treatment with the repositioned drugs loratadine and fluphenazine at low doses.

Finally, we also investigated whether loratadine could be used in combination with other antimitotic drugs. We tested eribulin, another antimitotic drug, which has been recently developed and used in the treatment of metastatic cancer (24-26). Previously, we found that KBV20C cell line is a very useful model to study highly eribulin-resistant cancer. As seen in Figure $4 \mathrm{~B}$ and $\mathrm{C}, 5 \mu \mathrm{M}$ of loratadine or fluphenazine produced similar sensitizing effects when combined with eribulin as compared to vincristineloratadine or vincristine-fluphenazine co-treatments (Figure 4A). Eribulin-verapamil co-treatment at the same dose also had effects similar to vincristine-verapamil co-treatment (Figure 4A and B). These results demonstrate that loratadine and fluphenazine are also effective in sensitizing resistant cancer cells to co-treatment with eribulin as much as vincristine. This finding also suggests that the histamine receptor antagonists loratadine and fluphenazine at low doses can be combined with other antimitotic drugs to sensitize the P-gp overexpressing cancer cells. We conclude that loratadine or fluphenazine can be used in different types of drug-resistant cancer.

We identified loratadine and azelastine from among 21 histamine receptor antagonists as highly sensitizing P-gpoverexpressing resistant cancer cells to antimitotic drugs. Interestingly, loratadine can sensitize them without any P-gp-inhibitory activity.

\section{Discussion}

In the current study, we investigated the novel application of some repositioned drugs for sensitizing P-gp-overexpressing drug-resistant cancer cells. Drug repositioning or drug repurposing is the application of known drugs for new indications. This strategy has been used for the treatment of various diseases and has advantages, such as low cost and avoidance of a significant number of toxicity tests, which is a time-consuming process (9-11). The urgent need for pharmacological treatments for resistant cancer can be efficiently addressed with drug repositioning and these drugs can be applied to patients at a relatively faster pace. We have previously investigated and suggested the use of antimalarial or anti-psychotic drugs against $\mathrm{P}$-gp-overexpressing resistant cancer cells $(12,13,20-22)$.

Previously, we found P-gp-overexpressing KBV20C cancer cells, which were highly resistant to antimitotic drugs, were sensitized by co-treatment with the repositioned drug fluphenazine, a histamine receptor antagonist $(12,13)$. As histamine receptor antagonists are located in the cellular membrane and contribute to inhibiting membrane-bound receptors $(15,16)$, we assumed that histamine receptor antagonists play a key role in reducing or modifying $\mathrm{P}-\mathrm{gp}$ activity in the membrane of resistant cancer cells. Based on a literature search, we found 21 other histamine receptor antagonists which are already used in the clinic. We then tested and evaluated these antagonists to identify novel application of these repositioned drugs in sensitizing P-gpoverexpressing resistant KBV20C cancer cells.

Most importantly, we identified two histamine receptor antagonists, loratadine and azelastine as being able to sensitize resistant KBV20C cells at a relatively lower dose than other histamine receptor antagonists. Considering that only two drugs (loratadine and azelastine) among the 21 antagonists were found to highly sensitize resistant cancer cells to vincristine, we can assume that sensitization of $\mathrm{P}$-gp-overexpressing resistant cancer cells is independent of histamine receptor inhibitory function. Although co-treatment with clemastine also showed vincristine-sensitizing effects on KBV20C cells, a much higher dose was needed than that required for loratadine or azelastine. Further mechanistic studies were focused on loratadine and azelastine co-treatment. Although the sensitizing effects of histamine receptor antagonists have been demonstrated, to our knowledge, our results are the first to reveal low dose of loratadine or azelastine as repositioned drugs. Considering that aging with allergic diseases is highly correlated with a higher incidence of cancer $(14,27,28)$, our findings might also contribute to selecting specific histamine receptor antagonists for preventing or reducing the incidence of cancer in elderly adults with allergic diseases. For example, anti-allergic drugs functioning in prevention of cancer might be used.

Our results were not limited to vincristine co-treatment since we found out that loratadine and azelastine had sensitizing effects against P-gp-overexpressing KBV20C cells, similar to those observed with eribulin. Eribulin, a recently developed agent, is promising for the treatment of resistant cancer (2426). We reported that P-gp-overexpressing KBV20C cells are 
highly eribulin-resistant $(12,23)$, and loratadine and fluphenazine can sensitize KBV20C cells to eribulin, suggesting that loratadine or fluphenazine might also sensitize cancer cells resistant to antimitotic and other type drugs.

A detailed analysis was performed to determine the molecular mechanisms underlying the sensitizing effects. We demonstrated that vincristine combined with loratadine or azelastine reduced cell proliferation and increased $\mathrm{G}_{2}$ arrest in the P-gp-overexpressing resistant KBV20C cells. Based on the microscopic observations, FACS results, and annexin $\mathrm{V}$ analyses, we concluded that apoptosis was increased by these co-treatments via increased $\mathrm{G}_{2}$ arrest and reduced proliferation. Loratadine showed much higher potential for $\mathrm{G}_{2}$ arrest and apoptotic cell death in combination with vincristine than did azelastine, confirming that loratadine is a better combination drug for the treatment of antimitotic drug-resistant cancer.

A repositioned drug fluphenazine, a histamine receptor antagonist, at a low dose, was shown to sensitize KBV20C cells $(12,13)$. Therefore, we determined whether loratadine or azelastine was able to sensitize KBV20C cells as much as fluphenazine. We found that loratadine, azelastine, and fluphenazine, have similar sensitizing effects at similar doses. This suggests that loratadine and azelastine can be considered as novel repositioned drugs having similar effects to the well-known repositioned drug fluphenazine.

As the efflux of vincristine by $\mathrm{P}$-gp is the main mechanism for resistance of $\mathrm{KBV} 20 \mathrm{C}$ cells to vincristine, we tested whether cell-sensitizing by loratadine or azelastine co-treatment resulted from the $\mathrm{P}$-gp-inhibitory effects of loratadine or azelastine. We demonstrated that azelastine had a high P-gpinhibitory activity similar to verapamil, suggesting that vincristine-azelastine sensitizing was the result of the effects of azelastine, which prevents pumping out of vincristine. However, interestingly, we did not detect any substantial P-gpinhibitory activity of loratadine, suggesting that loratadine removes or inhibits factors that block vincristine effects on drug-resistant cancer cells and that vincristine-loratadine then exerts a synergistic effect on co-treated cells. Further investigation with loratadine may be needed for determining the molecular targets which lead to sensitizing resistant cancer cells without P-gp inhibition. As no increased P-gp inhibition was detected with loratadine, an improved combination of chemotherapeutic agents can be developed for cancer patients who develop resistance to antimitotic drugs. As P-gp inhibitors are toxic to normal cells $(5,7)$, we assume that loratadine might be considered as a combination drug with a non-P-gp inhibitor to sensitize P-gp overexpressing resistant cancer cells. As personalized medicines are gaining popularity, our findings for the histamine receptor antagonists fluphenazine, loratadine, and azelastine might contribute to effective prescriptions in patients with drug-resistant cancer who are allergic or sensitive to the P-gp-inhibitory effect on normal tissues.
Taken together, the present results highlight the novel selective sensitizing effect of histamine receptor antagonists. Furthermore, drug-resistant KBV20C cells that overexpress P-gp can be sensitized to the antimitotic drugs eribulin or vincristine by co-treatment with the repositioned drugs fluphenazine, loratadine, and azelastine at low doses. Notably, loratadine sensitizes drug-resistant cancer cells without showing P-gp-inhibitory activity. Since the toxicities of these drugs have already been documented, they are readily available for clinical use. The present results may contribute to the improvement of the efficacy of various chemotherapeutic agents used alone or in combination for the treatment of patients with cancer which develops resistance to chemotherapeutic drugs via P-gp overexpression.

\section{Conflicts of Interest}

The Authors declare no conflicts of interest regarding this study.

\section{Authors' Contributions}

JYK: Collected the data, contributed data or analysis tools, wrote the article. KSK: collected the data, wrote the article. ISK: contributed data or analysis tools. SY: conceived and designed the analysis, collected the data. contributed data or analysis tools, wrote the article.

\section{Acknowledgements}

This research was supported by National Research Foundation of Korea (NRF) funded by the Ministry of Education (NRF2017R1D1A1B03029158).

\section{References}

1 Jordan MA and Wilson L: Microtubules as a target for anticancer drugs. Nat Rev Cancer 4: 253-265, 2004. PMID: 15057285. DOI: $10.1038 / \mathrm{nrc} 1317$

2 Kim JH, Yoo HI, Kang HS, Ro J and Yoon S: Salinomycin sensitizes antimitotic drugs-treated cancer cells by increasing apoptosis via the prevention of $\mathrm{G}_{2}$ arrest. Biochem Biophys Res Commun 418: 98-103, 2012. PMID: 22244892. DOI: 10.1016/ j.bbrc.2011.12.141

3 McGrogan BT, Gilmartin B, Carney DN and McCann A: Taxanes, microtubules and chemoresistant breast cancer. Biochim Biophys Acta 1785: 96-132, 2008. PMID: 18068131. DOI: $10.1016 /$ j.bbcan.2007.10.004

4 Szakacs G, Paterson JK, Ludwig JA, Booth-Genthe C and Gottesman MM: Targeting multidrug resistance in cancer. Nat Rev Drug Discov 5: 219-234, 2006. PMID: 16518375. DOI: $10.1038 /$ nrd1984

5 Chen Z, Shi T, Zhang L, Zhu P, Deng M, Huang C, Hu T, Jiang $\mathrm{L}$ and $\mathrm{Li} \mathrm{J}$ : Mammalian drug efflux transporters of the ATP binding cassette $(\mathrm{ABC})$ family in multidrug resistance: A review of the past decade. Cancer Lett 370: 153-164, 2016. PMID: 26499806. DOI: 10.1016/j.canlet.2015.10.010 
6 Chufan EE, Kapoor $\mathrm{K}$ and Ambudkar SV: Drug-protein hydrogen bonds govern the inhibition of the ATP hydrolysis of the multidrug transporter P-glycoprotein. Biochem Pharmacol 101: 40-53, 2016. PMID: 26686578. DOI: 10.1016/j.bcp. 2015.12.007

7 Shukla S, Wu CP and Ambudkar SV: Development of inhibitors of ATP-binding cassette drug transporters: Present status and challenges. Expert Opin Drug Metab Toxicol 4: 205-223, 2008. PMID: 18248313. DOI: 10.1517/17425255.4.2.205

8 Yang K, Wu J and Li X: Recent advances in the research of Pglycoprotein inhibitors. Biosci Trends 2: 137-146, 2008. PMID: 20103919.

9 Clark KB: New therapeutic bearings for repositioned drugs. Curr Top Med Chem 13: 2281-2282, 2013. PMID: 24059466.

10 Yoon S: A single treatment of selenate, a repositioning drug, specifically sensitizes P-gp-overexpressing resistant cancer cells. Cancer Cell Microenv 2(4), 2015. DOI: 10.14800/ccm.957

11 Pantziarka P and Cairns L: Recycling existing drugs for cancer therapy: delivering low cost cancer care. Ecancer Med Sci 8: ed40, 2014. PMID: 25075221. DOI: 10.3332/ecancer.2014.ed40

12 Cheon JH, Lee BM, Kim HS and Yoon S: Highly halavenresistant KBV20C cancer cells can be sensitized by co-treatment with fluphenazine. Anticancer Res 36: 5867-5874, 2016. PMID: 27793910. DOI: 10.21873/anticanres.11172

13 Kim JY, Park Y, Lee BM, Kim HS and Yoon S: P-gp Inhibition by the anti-psychotic drug pimozide increases apoptosis, as well as expression of $\mathrm{pRb}$ and $\mathrm{pH} 2 \mathrm{AX}$ in highly drug-resistant KBV20C cells. Anticancer Res 38: 5685-5692, 2018. PMID: 30275188. DOI: 10.21873 /anticanres.12905

14 Bachert C: A review of the efficacy of desloratadine, fexofenadine, and levocetirizine in the treatment of nasal congestion in patients with allergic rhinitis. Clin Ther 31: 921944, 2009. PMID: 19539095. DOI: 10.1016/j.clinthera.2009.05.

15 Ciprandi G, Tosca MA, Cosentino C, Riccio AM, Passalacqua $\mathrm{G}$ and Canonica GW: Effects of fexofenadine and other antihistamines on components of the allergic response: Adhesion molecules. J Allergy Clin Immunol 112: S78-82, 2003. PMID: 14530792.

16 Simons FE and Simons KJ: Clinical pharmacology of new histamine H1 receptor antagonists. Clin Pharmacokinet 36: 329352, 1999. PMID: 10384858. DOI: 10.2165/00003088-19993 6050-00003

17 Cheon JH, Kim JY, Lee BM, Kim HS and Yoon S: P-gp Inhibition by XL019, a JAK2 inhibitor, increases apoptosis of vincristine-treated resistant KBV20C cells with increased p21 and pH2AX expression. Anticancer Res 37: 6761-6769, 2017. PMID: 29187454. DOI: 10.21873/anticanres.12136

18 Cheon JH, Kim KS, Yadav DK, Kim M, Kim HS and Yoon S: The JAK2 inhibitors CEP-33779 and NVP-BSK805 have high P-gp-inhibitory activity and sensitize drug-resistant cancer cells to vincristine. Biochem Biophys Res Commun 490: 1176-1182, 2017. PMID: 28669723. DOI: 10.1016/j.bbrc.2017.06.178
19 Kim JY, Son JY, Lee BM, Kim HS and Yoon S: Aging-related repositioned drugs, donepezil and sildenafil citrate, increase apoptosis of anti-mitotic drug-resistant KBV20C cells through different molecular mechanisms. Anticancer Res 38: 5149-5157, 2018. PMID: 30194162. DOI: 10.21873/anticanres.12837

20 Kim JY, Tae IH, Lee BM, Kim HS and Yoon S: Low doses of the anti-psychotic drug aripiprazole have strong P-gp-inhibitory activity and sensitize anti-mitotic drug-resistant cancer cells. Anticancer Res 38: 5101-5108, 2018. PMID: 30194155. DOI: 10.21873/anticanres. 12830

21 Choi AR, Kim JH, Cheon JH, Kim HS and Yoon S: Attenuation of colchicine toxicity in drug-resistant cancer cells by cotreatment with anti-malarial drugs. Anticancer Res 36: 58595866, 2016. PMID: 27793909. DOI: 10.21873/anticanres.11171

22 Choi AR, Kim JH, Woo YH, Kim HS and Yoon S: Anti-malarial drugs primaquine and chloroquine have different sensitization effects with anti-mitotic drugs in resistant cancer cells. Anticancer Res 36: 1641-1648, 2016. PMID: 27069141.

23 Park Y, Son JY, Lee BM, Kim HS and Yoon S: Highly eribulinresistant KBV20C oral cancer cells can be sensitized by cotreatment with the third-generation P-glycoprotein inhibitor, elacridar, at a low dose. Anticancer Res 37: 4139-4146, 2017. PMID: 28739698. DOI: 10.21873/anticanres.11801

24 Dell'Ova M, De Maio E, Guiu S, Roca L, Dalenc F, Durigova A, Pinguet F, Bekhtari K, Jacot W and Pouderoux S: Tumour biology, metastatic sites and taxanes sensitivity as determinants of eribulin mesylate efficacy in breast cancer: results from the ERIBEX retrospective, international, multicenter study. BMC Cancer 15: 659, 2015. PMID: 26449988. DOI: 10.1186/s12885015-1673-3

25 Dybdal-Hargreaves NF, Risinger AL and Mooberry SL: Eribulin mesylate: mechanism of action of a unique microtubule-targeting agent. Clin Cancer Res 21: 2445-2452, 2015. PMID: 25838395. DOI: $10.1158 / 1078-0432 . C C R-14-3252$

26 Inoue K, Saito T, Okubo K, Kimizuka K, Yamada H, Sakurai T, Ishizuna K, Hata S, Kai T and Kurosumi M: Phase II clinical study of eribulin monotherapy in Japanese patients with metastatic breast cancer who had well-defined taxane resistance. Breast Cancer Res Treat 157: 295-305, 2016. PMID: 27125669. DOI: $10.1007 / \mathrm{s} 10549-016-3808-x$

27 D'Arcy M, Rivera DR, Grothen A and Engels EA: Allergies and the subsequent risk of cancer among elderly adults in the United States. Cancer Epidemiol Biomarkers Prev 28: 741-750, 2019. PMID: 30700443. DOI: 10.1158/1055-9965.EPI-18-0887

28 Karim AF, Westenberg LEH, Eurelings LEM, Otten R and Gerth van Wijk R: The association between allergic diseases and cancer: A systematic review of the literature. Neth J Med 77: 4266, 2019. PMID: 30895928.

Received April 9, 2019

Revised May 7, 2019

Accepted May 8, 2019 